\title{
Behandlung neu diagnostizierter Neuroblastome nach Ende der Klinischen Studien
}

\section{Treatment of newly diagnosed Neuroblastoma after the end of clinical trials}

\author{
Korrespondenzadresse \\ Prof. Dr. Jochen Rößler \\ Pädiatrische Hämatologie und Onkologie \\ Zentrum für Kinder- und Jugendmedizin \\ Universitätsklinikum Freiburg \\ Mathildenstr. 1, 79106 Freiburg \\ jochen.roessler@uniklinik-freiburg.de
}

\author{
Bibliografie \\ DOI http://dx.doi.org/10.1055/s-0043-106756 \\ Klin Padiatr 2017; 229: 111-112 \\ (c) Georg Thieme Verlag KG Stuttgart · New York \\ ISSN 0300-8630
}

Liebe Leserinnen und Leser,

Anfang Dezember 2016 bedankte sich Herr Prof. Frank Berthold in einem Schreiben an die GPOH Neuroblastom-Studienzentren ich zitiere - „...für 37 exzellente Jahre Kooperation für Patienten mit Neuroblastomen aller Ausprägungsgrade in Deutschland und einer Gesamt-Einschlussrate von $97 \%$ in 6 lückenlos aufeinander folgende Studien“. Anlass war das Ende der Rekrutierung für die Studie NB-2004 HR zum 31.12.2016. Auch die Studie NB-2004 endete zu diesem Zeitpunkt.

Seit Beginn des Jahres 2017 ist nun anscheinend eine „Lücke“ entstanden ...

Prinzipiell soll nun die Behandlung von Neuroblastom Patienten nach der bisherigen, in den langen Jahren der gemeinsamen Zusammenarbeit und Rekrutierung erarbeiteten Diagnostik und Standardtherapie erfolgen. Die entsprechenden Algorithmen für Diagnostik, Risikostratifizierung und Therapie sind im Artikel „2017 GPOH Guidelines for Diagnosis and Treatment of Patients with Neuroblastic Tumors“ von Seite 147 bis 167 in dieser Ausgabe zu finden. Dies hatten die aktuellen Neuroblastom Studienleitern in einem Schreiben Anfang Januar 2017 bereits den Zentren angekündigt.

In Deutschland sind von 1980 bis 2014 insgesamt 4172 Neuroblastom Erkrankungen unter 15 Jahre im Kinderkrebsregister gemeldet worden. In derselben Quelle wird die 5-Jahres-Überlebenswahrscheinlichkeit mit aktuell $79 \%$ angegeben (Jahresbericht des Kinderkrebsreigsters aus dem Jahr 2015). Sie hatte sich stetig über die Jahre hinweg deutlich verbessert ( $>$ Abb. 1). Dies ist der Verdienst der oben genannten „exzellenten Jahre Kooperation ... mit einer Gesamt-Einschlussrate von $97 \%$..."

Der hier veröffentlichte Artikel wird helfen, Patienten mit Neuroblastomen nach dem aktuellen Wissensstand zu diagnostizieren und zu behandeln. Es ist jedoch keine Frage, dass die aktuelle „Lücke“ so schnell wie möglich geschlossen werden sollte und neue klinische Studien mit innovativen Therapieansätzen gestartete werden müssen, um den Erfolg der Behandlung noch zu steigern.

Warum war es denn „früher“ möglich „... 6 lückenlos aufeinander folgende Studien...“ zu realisieren und zu keinem Zeitpunkt während „....37 Jahren...“ eine Lücke entstehen zu lassen? Die Dis- kussion in diesem Bereich ist sehr intensiv und in unserer Fachgesellschaft zu recht häufig kontrovers: werden heute Regularien, die für Europa gelten und national umgesetzt werden müssen, zu „Stolpersteinen“ und „Hindernissen“ für die Durchführung von klinischen Studien? Ist das AMG, die oft mit Stirnrunzeln ausgesprochene Abkürzung für das Arzneimittelgesetzt, ein „rotes Tuch“ für die „ückenlose“ und erfolgreiche Arbeit in der Pädiatrischen Hämatologie und Onkologie? Die Daten zur Diagnostik und Behandlung von Neuroblastompatienten gehen in der Zeit ohne klinische Studien zum Glück nicht verloren: dafür sorgen „Register“, wie dies auch in vielen anderen Krankheitsentitäten in unserem Fachgebiet gehandhabt wird. Das Neuroblastom Register-2016 für Neuroblastome aller Altersstufen, Stadien und Rezidive sowie das Register für Neuroblastische Tumoren mit intraspinalen Anteilen (NB with $\mathrm{SCl}$ registry) sind beide seit kurzem aktiv. Die Ergebnisse und Ereignisse während der Therapie von Neuroblastomen werden durch diese Register erfasst. Eine systematische und wissenschaftlich Durchfüh-

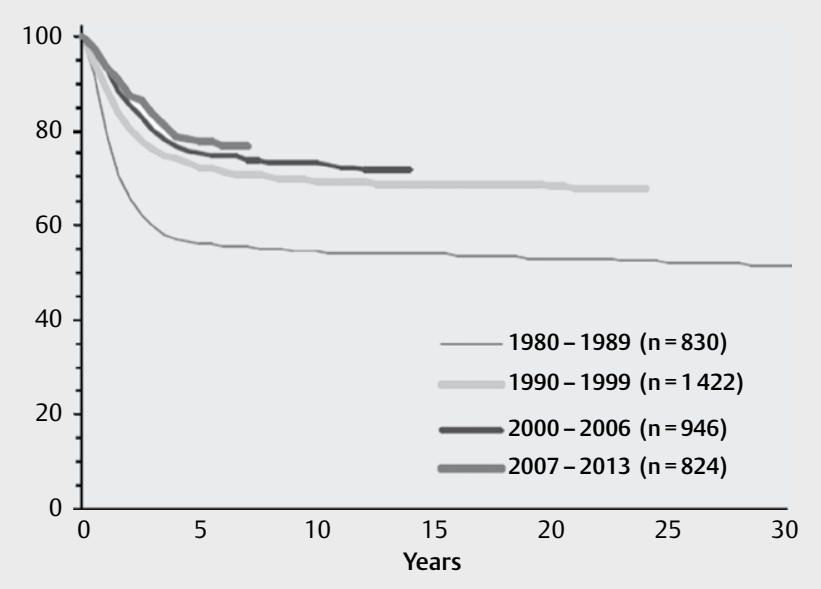

- Abb. 1 Überlebenswahrscheinlichkeit nach Diagnosejahr in Deutschland von 1980-2013 (aus dem Jahresbericht des Kinderkrebsregisters 2015) 
rung der Behandlung wird hingegen dadurch nicht garantiert. Hierzu bedarf es der klinischen Studien: klinische Studien der Phase II oder III im Bereich der Ersterkrankungen. Die Durchführung dieser Projekte unterliegt entsprechenden Regularien, dem AMG Gesetz. Das bedeutet einen extremen Arbeitsaufwand für die Teams aus Studienleiter, Koordinatoren, Prüfärzten, Studynurses und Dokumentaren. So entstehen Kosten, die nicht durch Gelder der Krankenversorgung und der zusätzlichen Projektförderung abgedeckt werden können. Wie soll mit dieser Problematik umgegangen werden?

Ich bin überzeugt, dass es internationale Zusammenarbeit bedarf und Aufklärungsarbeit, um in der Bevölkerung und damit in der Öffentlichkeit und Politik für diese Thematik Interesse zu finden. Die SIOP-Europe ist mit all Ihren Parnterorganisation auf einem guten Weg, die Stimme der betroffenen Patienten, ihrer Familien, der betreuenden Ärzte und Forscher lauter und hörbarer zu machen (siehe z. B. die ACCELERATW Plattform - http://www.accelerateplatform.eu/). Es wird noch eine Zeit dauern, bis diese Aktionen mehr Früchte tragen. In den verschiedenen Studiengruppen und in der GPOH Fachgesellschaft sollte die Diskussion aufgenommen und fortgesetzt werden.

Die Neuroblastom Studienleiter teilten mit, dass zurzeit 2 klinische Studien nach AMG für Neuroblastompatienten in Vorbereitung sind: eine Studie für „Low Risk“ und „Intermediate Risk“-Patienten, die aktuell zur Prüfung bei der Ethikkommission Köln vorliegt. Eine weitere Studie für sowohl „High Risk“ Patienten und Rezidivpatienten ist auf europäischer Ebene unter dem Dach der SIOPEN Gruppe geplant. Bis zum Start dieser Projekte wird uns allen der Beitrag dieser Ausgabe der Klinischen Pädiatrie Unterstützung geben.

Interessenkonflikt

Der Autor erklärt, dass er innerhalb der vergangenen 3 Jahre in einem Beratungsgremium von Pierre Fabre tätig war und dass er von der Firma Pfizer Forschungsunterstützung erhalten hat. 